\title{
Volterra operators between limits of Bergman-type weighted spaces of analytic functions
}

\author{
Ersin Kizgut (15) \\ Instituto Universitario de Matemática Pura y Aplicada (IUMPA), Universitat Politècnica de València, \\ E-46071 Valencia, Spain
}

\begin{abstract}
We characterize continuity and compactness of the Volterra integral operator $T_{g}$ with the non-constant analytic symbol $g$ between certain weighted Fréchet or (LB)-spaces of analytic functions on the open unit disc, which arise as projective (resp. inductive) limits of intersections (resp. unions) of Bergman spaces of order $1<p<\infty$ induced by the standard radial weight $\left(1-|z|^{2}\right)^{\alpha}$ for $0<\alpha<\infty$. Motivated from the earlier results obtained for weighted Bergman spaces of standard weight, we also establish several results concerning the spectrum of the Volterra operators acting on the weighted Bergman Fréchet space $A_{\alpha+}^{p}$, and acting on the weighted Bergman (LB)-space $A_{\alpha-}^{p}$.
\end{abstract}

Mathematics Subject Classification (2020). 47A10, 47B38, 46A11, 46E10, 47A35, $47 \mathrm{~B} 10$

Keywords. Volterra operator, weighted spaces of analytic function, Bergman spaces, Fréchet spaces, (LB)-spaces, spectrum

\section{Introduction}

The aim of this note is to investigate continuity, compactness and spectrum of the Volterra integral operator defined on certain weighted Fréchet or (LB)-spaces of analytic functions on the open unit disc $\mathbb{D}$ of the complex plane. For a non-constant analytic function $g \in H(\mathbb{D})$, the Volterra operator $T_{g}$ with symbol $g$ is defined on the space $H(\mathbb{D})$ of analytic functions on the unit disc by

$$
T_{g}(f)(z):=\int_{0}^{z} f(\zeta) g^{\prime}(\zeta) \mathrm{d} \zeta, \quad z \in \mathbb{D} .
$$

We investigate the Volterra operator $T_{g}$ when it is defined on spaces which appear as intersections or unions of the weighted Bergman spaces of analytic functions described below. The Bergman space $A_{\alpha}^{p}=A_{\alpha}^{p}(\mathbb{D})$ of order $1<p<\infty$ induced by the standard radial weight $\left(1-|z|^{2}\right)^{\alpha}$ for $-1<\alpha<\infty$ is given by

$$
A_{\alpha}^{p}:=\left\{f \in H(\mathbb{D}):\|f\|_{p, \alpha}=\left((\alpha+1) \int_{\mathbb{D}}|f(z)|^{p} \mathrm{~d} s_{\alpha}(z)\right)^{1 / p}<\infty\right\},
$$

Email address: erkiz@upv.es

Received: 07.08.2020; Accepted: 02.02.2021 
$\mathrm{d} s_{\alpha}(z)=\left(1-|z|^{2}\right)^{\alpha} \mathrm{d} s(z)$, and $\mathrm{d} s(z)=\frac{1}{\pi} \mathrm{d} x \mathrm{~d} y$. One might also use the weight $(1-|z|)^{\alpha}$ instead of $\left(1-|z|^{2}\right)^{\alpha}$. Since $1-|z| \leq 1-|z|^{2} \leq 2(1-|z|)$, these weights induce the same space and the norms are equivalent. Each $A_{\alpha}^{p}$ is a closed subspace of $L^{p}(\mathbb{D}, \mathrm{d} s(z))$ in which the polynomials are dense [15, Section 1.1]. The weighted Bergman space $A_{\alpha}^{p}$ is a Banach space with the norm $\|\cdot\|_{p, \alpha}$. Classical Bergman space $A^{p}(\mathbb{D})$ corresponds to the case $\alpha=0$. We denote by $A_{\omega}^{p}$ a weighted Bergman space of order $1<p<\infty$ with the weight function $\omega(z)$ such that it becomes a Banach space with the norm

$$
\|f\|_{p, \omega}:=\left(\int_{\mathbb{D}}|f(z)|^{p} \omega(z) \mathrm{d} s(z)\right)^{1 / p} .
$$

The aim of this paper is to investigate the Volterra operator $T_{g}$ on spaces that arise as intersections and unions of Bergman spaces of order $1<p<\infty$ induced by the standard radial weights $\left(1-|z|^{2}\right)^{\alpha}$ for $0<\alpha<\infty$. The weighted Bergman Fréchet space $A_{\alpha+}^{p}$ is given by

$$
\begin{aligned}
A_{\alpha+}^{p} & :=\left\{f \in H(\mathbb{D}):\left(\int_{\mathbb{D}}|f(z)|^{p} \mathrm{~d} s_{\mu}(z)\right)^{1 / p}<\infty, \forall \mu>\alpha\right\} \\
& =\bigcap_{\mu>\alpha} A_{\mu}^{p}=\bigcap_{n \in \mathbb{N}} A_{\left(\alpha+\frac{1}{n}\right)}^{p}=\operatorname{proj}_{n \in \mathbb{N}} A_{\left(\alpha+\frac{1}{n}\right)}^{p}
\end{aligned}
$$

The weighted Bergman (LB)-space $A_{\alpha-}^{p}$ is defined by

$$
\begin{aligned}
A_{\alpha-}^{p} & :=\left\{f \in H(\mathbb{D}):\left(\int_{\mathbb{D}}|f(z)|^{p} \mathrm{~d} s_{\mu}(z)\right)^{1 / p}<\infty, \text { for some } \mu<\alpha\right\} \\
& =\bigcup_{\mu<\alpha} A_{\mu}^{p}=\bigcup_{n \in \mathbb{N}} A_{\left(\alpha-\frac{1}{n}\right)}^{p}=\operatorname{ind}_{n \in \mathbb{N}} A_{\left(\alpha-\frac{1}{n}\right)}^{p},
\end{aligned}
$$

where the inductive limit is taken over all $n \in \mathbb{N}$ such that $\left(\alpha-\frac{1}{n}\right)>0$. The monograph [15] presents an investigation of Bergman type spaces (for $p=\infty$ ) in that fashion with relevance to interpolation and sampling of analytic functions. The paper [18] gives a description of intersections and unions of weighted Bergman spaces of order $0<p<\infty$. Unlike those, we treat the space $A_{\alpha+}^{p}$ as a Fréchet space when equipped with the locally convex topology generated by the increasing system of norms

$$
\|f\|_{p, \alpha, n}:=\left(\int_{\mathbb{D}}|f(z)|^{p} \mathrm{~d} s_{\left(\alpha+\frac{1}{n}\right)}(z)\right)^{1 / p}
$$

for $f \in A_{\alpha+}^{p}$ and each $n \in \mathbb{N}$. Analogously, $A_{\alpha-}^{p}$ is an (LB)-space endowed with the finest locally convex topology such that each natural inclusion map from $A_{\mu}^{p}$ into $A_{\gamma}^{p}$, for $0<\mu<\gamma$ is continuous. It is also regular, since every bounded set $B \subseteq A_{\alpha-}^{p}$ is contained and bounded in the Banach space $A_{\mu}^{p}$, for some $0<\mu<\alpha$. We also note that, for $1<p<\infty$ and $0<\alpha<\infty$ we have $A_{\alpha-}^{p} \subset A_{\alpha}^{p} \subset A_{\alpha+}^{p}$, where each inclusion is continuous. Since for each pair $0<\mu<\gamma<\infty$ the canonical inclusion map $\iota: A_{\mu}^{p} \rightarrow A_{\gamma}^{p}$ is compact (see, for instance, [17, Proposition 3.1] for a proof), both $A_{\alpha+}^{p}$ and $A_{\alpha-}^{p}$ are Schwartz spaces by [16, §21.1, Example 1(b)] and [20, Proposition 25.20]. We characterize continuity and compactness of $T_{g}$ between different weighted Bergman Fréchet and (LB)spaces in Section 2. In connection with previous significant results obtained for Banach spaces, Section 3 is devoted to the spectral properties of $T_{g}$ acting on a weighted Bergman Fréchet or (LB)-space.

If $g(z)=z$, for $z \in \mathbb{D}$, then the Volterra operator coincides with the integration operator $J$. If we let $g(z)=-\log (1-z)$, for $z \in \mathbb{D}$, then $C(f)(z):=\frac{1}{z} T_{g}(f)(z), z \in \mathbb{D}, z \neq 0$, $\mathrm{C}(f)(0):=f(0)$ is the Cesàro operator. This operator acting on $A_{\alpha+}^{p}$ and $A_{\alpha-}^{p}$ was investigated by the author in [17]. 
The Volterra operator for holomorphic functions on the unit disc was introduced by Pommerenke [21]. He showed that $T_{g}$ is bounded on the Hardy space $H^{2}$ if and only if $g \in B M O A$. Aleman and Siskakis extended this result to $H^{p}, 1 \leq p<\infty$ [8]. Later they also considered the case of weighted Bergman spaces [9]. We also refer to [4,10]. Aleman and Constantin [5], Aleman and Persson [7], Aleman and Paláez [6] studied the spectra of Volterra and Cesàro operators on several spaces of holomorphic functions on the unit disc. For a survey regarding Volterra operators defined on several spaces of analytic functions, see [23].

The motivation of the present research is due to the investigation on the spectrum of Volterra-type operators on weighted Bergman spaces carried out by Aleman and Constantin in [5], and the study of the author [17] where continuity, compactness and spectrum of Cesàro operator in the same context of unions and intersections of weighted Bergman spaces considered here inspired by the works of Albanese, Bonet and Ricker [1,3]. The Volterra operator is closely related to Cesàro operator. Our results, however, requires different approaches and new ingredients. For an analogue study concerning the Volterra operator in limits of growth spaces of analytic functions, we refer to Bonet [12] in relation with [19].

\section{Continuous of Volterra operators on $A_{\alpha+}^{p}$ and $A_{\alpha-}^{p}$}

The Volterra operator $T_{g}$, where $g$ belongs to $H(\mathbb{D})$ is continuous on the weighted Bergman space $A_{\alpha}^{p}$ if and only if $g$ belongs to the Bloch space $\mathcal{B}_{1}$. This is proved in [9, Theorem 1]. Let us remind that a holomorphic function $g$ belongs to $\mathcal{B}_{\alpha}$ for $0 \leq \alpha<\infty$ if and only if $\sup _{z \in \mathbb{D}}\left|g^{\prime}(z)\right|\left(1-|z|^{2}\right)^{\alpha}<\infty$. The function $g \in H(\mathbb{D})$ belongs to the little Bloch space $\mathcal{B}_{\alpha}^{0} \subset \mathcal{B}_{\alpha}$ if and only if $\lim _{|z| \rightarrow 1^{-}}\left|g^{\prime}(z)\right|\left(1-|z|^{2}\right)^{\alpha}=0$. We refer the reader to [24] for further information on Bloch spaces. The following result is due to [22, Theorem 2]. It shall be quoted frequently in our proofs.

Proposition 2.1. Let $g \in H(\mathbb{D})$ be an analytic function. Suppose that $1<p, q<\infty$, and $-1<\alpha, \beta<\infty$.

(i) Let $p \leq q$ and $\frac{2+\alpha}{p}-\frac{2+\beta}{q} \leq 1$. Then, the Volterra operator $T_{g}: A_{\alpha}^{p} \rightarrow A_{\beta}^{q}$ is

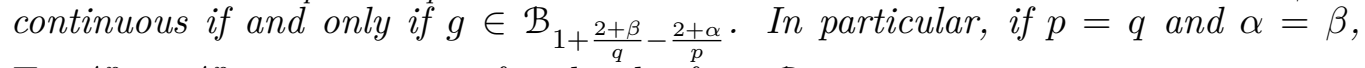
$T_{g}: A_{\alpha}^{p} \rightarrow A_{\alpha}^{p}$ is continuous if and only if $g \in \mathcal{B}_{1}$.

(ii) Let $q<p$ and $\frac{2+\alpha}{p}-\frac{2+\beta}{q} \leq 1$. Then, the Volterra operator $T_{g}: A_{\alpha}^{p} \rightarrow A_{\beta}^{q}$ is continuous if and only if $g^{\prime} \in A_{s+\gamma}^{s}$, where $\frac{1}{s}=\frac{1}{q}-\frac{1}{p}$ and $\gamma=\frac{p \beta-q \alpha}{p-q}$.

(iii) Let $\frac{2+\alpha}{p}-\frac{2+\beta}{q}>1$, and suppose that $T_{g}: A_{\alpha}^{p} \rightarrow A_{\beta}^{q}$ be continuous. Then, $g$ is constant.

Proposition 2.2. Let $1<p \leq q<\infty$ and $0<\alpha, \beta<\infty$ satisfy $\gamma:=1+\frac{2+\beta}{q}-\frac{2+\alpha}{p} \geq 0$. Let $g \in H(\mathbb{D})$ be an analytic function.

(1) The Volterra operator $T_{g}: A_{\alpha+}^{p} \rightarrow A_{\beta+}^{q}$ is continuous if and only if

$$
g \in \bigcap_{\tau \in(\gamma, x)} \mathcal{B}_{\tau}
$$

for every $x>\gamma$. In particular,

(i) The Volterra operator $T_{g}: A_{\alpha+}^{p} \rightarrow A_{\alpha+}^{p}$ is continuous if and only if

$$
g \in \bigcap_{\tau \in(1, x)} \mathcal{B}_{\tau}
$$

for every $x>1$.

(ii) Let $\frac{2+\alpha}{p} \leq \frac{2+\beta}{q}$. The Volterra operator $T_{g}: A_{\alpha+}^{p} \rightarrow A_{\beta+}^{q}$ is continuous if $g$ satisfies (2.2) for every $x>1$. 
(iii) Let $\frac{2+\beta}{q}<\frac{2+\alpha}{p}$. If the Volterra operator $T_{g}: A_{\alpha+}^{p} \rightarrow A_{\beta+}^{q}$ is continuous, then $g$ satisfies (2.2) for every $x>1$.

(2) The Volterra operator $T_{g}: A_{\alpha-}^{p} \rightarrow A_{\beta-}^{q}$ is continuous if and only if $g$ satisfies (2.1) for $x=\gamma+\min \left\{(\alpha+1) \frac{q-1}{p q},(\beta+1) \frac{q-1}{q}\right\}$. In particular,

(i) The Volterra operator $T_{g}: A_{\alpha-}^{p} \rightarrow A_{\alpha-}^{p}$ is continuous if and only if $g$ satisfies (2.2) for $x=1+\frac{\alpha+1}{2 p}$.

(ii) Let $\frac{2+\alpha}{p} \leq \frac{2+\beta}{q}$. The Volterra operator $T_{g}: A_{\alpha-}^{p} \rightarrow A_{\beta-}^{q}$ is continuous if $g$ satisfies (2.2) for $x=1+\frac{\alpha+1}{2 p}$.

(iii) Let $\frac{2+\beta}{q}<\frac{2+\alpha}{p}$. If the Volterra operator $T_{g}: A_{\alpha-}^{p} \rightarrow A_{\beta-}^{q}$ is continuous, then $g$ satisfies $(2.2)$ for $x=1+\frac{\min \{\alpha, \beta\}+1}{p}$.

Proof. (1) Since the polynomails are dense in each of $A_{\mu}^{p}$, for every $\mu>\alpha$, by (1.2), the Fréchet space $A_{\alpha+}^{p}$ is a reduced projective limit of the Banach spaces $A_{\mu}^{p}, \mu>\alpha$. This implies, $T_{g}: A_{\alpha+}^{p} \rightarrow A_{\beta+}^{q}$ is continuous if and only if for each $\mu>\beta$ there is $\alpha<\eta<$ $\alpha+\mu-\beta$ such that $T_{g}: A_{\eta}^{p} \rightarrow A_{\mu}^{q}$ is continuous. Now let $g$ satisfy (2.1) for every $x>\gamma$. For any $\varepsilon>0$, find $\delta \in(0, \varepsilon)$ such that $g \in \mathcal{B}_{\gamma+\varepsilon-\delta}$. Given $\mu:=\beta+q \varepsilon$, define $\eta:=\alpha+p \delta$ and let us observe that for every $z \in \mathbb{D}$,

$$
\left|g^{\prime}(z)\right|\left(1-|z|^{2}\right)^{1+\frac{2+\mu}{q}-\frac{2+\eta}{p}}=\left|g^{\prime}(z)\right|\left(1-|z|^{2}\right)^{\gamma+\varepsilon-\delta} .
$$

Hence $g \in \mathcal{B}_{1+\frac{2+\mu}{q}-\frac{2+\eta}{p}}$. So $T_{g}: A_{\eta}^{p} \rightarrow A_{\mu}^{q}$ is continuous by Proposition 2.1(i). Therefore $T_{g}: A_{\alpha+}^{p} \rightarrow A_{\beta+}^{q}$ is also continuous. For the converse, let $T_{g}: A_{\alpha+}^{p} \rightarrow A_{\beta+}^{q}$ be continuous. Then, for every $\varepsilon>0$, given $\mu:=\beta+q \varepsilon$ there exists $\alpha<\eta<\alpha+q \varepsilon$ such that $T_{g}: A_{\eta}^{p} \rightarrow A_{\mu}^{q}$ is continuous. By Proposition 2.1(i), $g \in \mathcal{B}_{1+\frac{2+\mu}{q}-\frac{2+\eta}{p}}$. Then, observe that for every $z \in \mathbb{D}$,

$$
\left|g^{\prime}(z)\right|\left(1-|z|^{2}\right)^{1+\frac{2+\mu}{q}-\frac{2+\eta}{p}}>\left|g^{\prime}(z)\right|\left(1-|z|^{2}\right)^{\gamma+\varepsilon} .
$$

Hence, $g \in \mathcal{B}_{\gamma+\varepsilon}$ for every $\varepsilon>0$. So (2.1) holds for all $x>\gamma$. In particular, if we let $p=q$ and $\alpha=\beta$, part (i) follows immediately. If we let $\frac{2+\alpha}{p} \leq \frac{2+\beta}{q}$, then by (2.3), part (ii) follows. If we suppose $\frac{2+\beta}{q}<\frac{2+\alpha}{p}$, then by (2.4), part (iii) holds.

(2) Let $x=\min \left\{(\alpha+1) \frac{q-1}{p q},(\beta+1) \frac{q-1}{q}\right\}$. By Grothendieck's factorization theorem (see e.g. [20, Theorem 24.33]), $T_{g}: A_{\alpha-}^{p} \rightarrow A_{\alpha-}^{p}$ is continuous if and only if for every $-1<\mu<\alpha$, there exists $\mu<\eta<\alpha$ such that $T_{g}: A_{\mu}^{p} \rightarrow A_{\eta}^{p}$ is continuous. Suppose that for every $\varepsilon \in(0, x)$ we have $g \in \mathcal{B}_{\gamma+\varepsilon}$. Then, given $-1<\mu:=\alpha-\frac{p q}{q-1} \varepsilon$ define $-1<\eta:=\beta-\frac{q}{q-1} \varepsilon$. Observe that for every $z \in \mathbb{D}$,

$$
\begin{aligned}
\left|g^{\prime}(z)\right|\left(1-|z|^{2}\right)^{1+\frac{2+\eta}{q}-\frac{2+\mu}{p}} & =\left|g^{\prime}(z)\right|\left(1-|z|^{2}\right)^{\gamma+\frac{q}{q-1} \varepsilon-\frac{1}{q-1} \varepsilon} \\
& =\left|g^{\prime}(z)\right|\left(1-|z|^{2}\right)^{\gamma+\varepsilon} .
\end{aligned}
$$

Thus, $g \in \mathcal{B}_{1+\frac{2+\eta}{q}-\frac{2+\mu}{p}}$. By Proposition 2.1(i), $T_{g}: A_{\mu}^{p} \rightarrow A_{\eta}^{q}$ is continuous. Then, $T_{g}: A_{\alpha-}^{p} \rightarrow A_{\beta-}^{q}$ is also continuous. For the converse, let $T_{g}: A_{\alpha-}^{p} \rightarrow A_{\beta-}^{q}$ be continuous. Then, for every $-1<\mu:=\alpha-p \varepsilon$ there exists $-1<\beta-p \varepsilon<\eta:=\beta-p \delta$ such that $T_{g}: A_{\mu}^{p} \rightarrow A_{\eta}^{q}$ is continuous. Then, by Proposition 2.1(i), $g \in \mathcal{B}_{1+\frac{2+\eta}{q}-\frac{2+\mu}{p}}$. Thus, for every $z \in \mathbb{D}$,

$$
\left|g^{\prime}(z)\right|\left(1-|z|^{2}\right)^{1+\frac{2+\eta}{q}-\frac{2+\mu}{p}}=\left|g^{\prime}(z)\right|\left(1-|z|^{2}\right)^{\gamma+\varepsilon-\frac{p}{q} \delta} .
$$

Hence, there exists $\hat{\delta} \in\left(0, \frac{p}{q} \varepsilon\right]$ such that $g \in \mathcal{B}_{\gamma+\varepsilon-\hat{\delta}}$. This happens when (2.1) is satisfied. In particular, if we let $p=q$ and $\alpha=\beta$, part (i) follows immediately. If we let $\frac{2+\alpha}{p} \leq \frac{2+\beta}{q}$, then by (2.5), part (ii) follows. If we suppose $\frac{2+\beta}{q}<\frac{2+\alpha}{p}$, then by (2.6) we get part (iii). 
Remark 2.3. Let $\gamma:=1+\frac{2+\beta}{q}-\frac{2+\alpha}{p} \geq 0$. Concerning Proposition 2.2, suppose that $g \in \bigcap_{\tau>\gamma} \mathcal{B}_{\tau} \backslash \mathcal{B}_{\gamma}$. Then, for every $\varepsilon>0$, we have $g \in \mathcal{B}_{\gamma+\varepsilon} \backslash \mathcal{B}_{\gamma}$. Then, given $\mu:=\beta+q \varepsilon$, for every $z \in \mathbb{D}$,

$$
\left|g^{\prime}(z)\right|\left(1-|z|^{2}\right)^{1+\frac{2+\mu}{q}-\frac{2+\alpha}{p}}=\left|g^{\prime}(z)\right|\left(1-|z|^{2}\right)^{\gamma+\varepsilon} .
$$

So, $g \in \mathcal{B}_{1+\frac{2+\mu}{q}-\frac{2+\alpha}{p}}$. Hence $T_{g}: A_{\alpha}^{p} \rightarrow A_{\mu}^{q}$ is continuous. Thus, the continuity estimate

$$
\left\|T_{g} f\right\|_{q, \beta+q \varepsilon} \lesssim\|f\|_{p, \alpha}, \quad f \in A_{\alpha+}^{p} .
$$

is optimal. That is, we cannot get rid of the term $q \varepsilon$, by the density argument we also used in Proposition 2.2.

The following proposition directly follows from Proposition 2.1(ii).

Proposition 2.4. Let $1<q<p<\infty$ and $0<\alpha, \beta<\infty$ satisfy $\frac{2+\alpha}{p}-\frac{2+\beta}{q} \leq 1$. Define $\frac{1}{s}=\frac{1}{q}-\frac{1}{p}$. Then,

(1) The Volterra operator $T_{g}: A_{\alpha+}^{p} \rightarrow A_{\beta+}^{q}$ is continuous if and only if for every $\varepsilon>0$ there exists $\delta \in(0, \varepsilon]$ such that $g^{\prime} \in A_{s+\gamma}^{s}$, where $\gamma=\frac{p(\beta+\varepsilon)-p(\alpha+\delta)}{p-q}$.

(2) The Volterra operator $T_{g}: A_{\alpha-}^{p} \rightarrow A_{\beta-}^{q}$ is continuous if and only if for every $\varepsilon \in(0, \beta+1)$ there exists $\delta \in(0, \varepsilon]$ such that $g^{\prime} \in A_{s+\mu}^{s}$, where $\mu=\frac{p(\beta-\delta)-p(\alpha-\varepsilon)}{p-q}$.

An operator $T: X \rightarrow X$ on a space $X$ is called compact (resp. bounded) if there exists a zero neighborhood $U$ such that $T(U)$ is a relatively compact (resp. bounded) subset of $X$. If the bounded subsets of $X$ are relatively compact, i.e., $X$ is a Schwartz space, then bounded operators coincides with compact operators. By [17, Corollary 3.2], this is the case for $A_{\alpha+}^{p}$ and $A_{\alpha-}^{p}$. Therefore, Proposition 2.1 will play a crucial role in characterizing compactness of the Volterra operator $T_{g}$ on $A_{\alpha+}^{p}$ and $A_{\alpha-}^{p}$. The following well-known abstract lemma will help us prove our main result on compactness.

Lemma 2.5. (i) Let $E=\operatorname{proj}_{m} E_{m}$ and $F=\operatorname{proj}_{n} F_{n}$ be Fréchet spaces such that $E$ (resp. F) is the intersection of the sequence of Banach spaces $E_{m}$ (resp. $F_{n}$ ), E is dense in $E_{m}$ and $E_{m+1} \subset E_{m}$ with continuous inclusion for each $m$ (resp. $F$ is dense in $F_{n}$ and $F_{n+1} \subset F_{n}$ with continuous inclusion for each $\left.n\right)$. Let $T: E \rightarrow F$ be a linear operator. Assume $T$ is continuous. Then $T$ is bounded if and only if there is $m$ such that for each $n$, $T$ has a unique continuous linear extension $T_{m, n}: E_{m} \rightarrow F_{n}$.

(ii) Let $X=\operatorname{ind} X_{n}$ and $Y=\operatorname{ind} Y_{m}$ be two (LB)-spaces which are increasing unions of Banach spaces $X=\cup_{n=1}^{\infty} X_{n}$ and $Y=\cup_{m=1}^{\infty} Y_{m}$. Let $T: X \rightarrow Y$ be a continuous linear map. Assume that $Y$ is a regular (LB)-space. Then $T$ is bounded if and only if there exists $m \in \mathbb{N}$ such that $T\left(X_{n}\right) \subset Y_{m}$ and $T: X_{n} \rightarrow Y_{m}$ is continuous for all $n \geq m$.

Proposition 2.6. Let $1<p \leq q<\infty$, and $0<\alpha, \beta<\infty$ satisfy $\gamma:=1+\frac{2+\beta}{q}-\frac{2+\alpha}{p}>0$. Let $g \in H(\mathbb{D})$ be an analytic function.

(1) The Volterra operator $T_{g}: A_{\alpha+}^{p} \rightarrow A_{\beta+}^{q}$ is compact if and only if

$$
g \in \bigcup_{\tau<\gamma} \mathcal{B}_{\tau} .
$$

In particular,

(i) The Volterra operator $T_{g}: A_{\alpha+}^{p} \rightarrow A_{\alpha+}^{p}$ is compact if and only if

$$
g \in \bigcup_{\tau<x} \mathcal{B}_{\tau}
$$

for $x=1$. 
(ii) Let $\frac{2+\alpha}{p} \leq \frac{2+\beta}{q}$. Then, the Volterra operator $T_{g}: A_{\alpha+}^{p} \rightarrow A_{\beta+}^{q}$ is compact if $g$ satisfies (2.9) for $x=1$.

(iii) Let $\frac{2+\beta}{q}<\frac{2+\alpha}{p}$. If the Volterra operator $T_{g}: A_{\alpha+}^{p} \rightarrow A_{\beta+}^{q}$ is compact, then $g$ satisfies (2.9) for $x=1$.

(2) The Volterra operator $T_{g}: A_{\alpha_{-}}^{p} \rightarrow A_{\beta-}^{q}$ is compact if and only if $g$ satisfies (2.8). In particular,

(i) The Volterra operator $T_{g}: A_{\alpha-}^{p} \rightarrow A_{\alpha-}^{p}$ is compact if and only if $g$ satisfies (2.9) for $x=\min \left\{1, \frac{\alpha+1}{p}\right\}$.

(ii) Let $\frac{2+\beta}{q}<\frac{2+\alpha}{p}$. If the Volterra operator $T_{g}: A_{\alpha-}^{p} \rightarrow A_{\beta-}^{q}$ is compact, then $g$ satisfies (2.9) for $x=\frac{\min \{\alpha, \beta\}+1}{q}$.

(iii) Let $\frac{2+\alpha}{p} \leq \frac{2+\beta}{q}$. The Volterra operator $T_{g}: A_{\alpha-}^{p} \rightarrow A_{\beta-}^{q}$ is compact if $g$ satisfies (2.9) for $x=\frac{\alpha+1}{q}$.

Proof. (1) Suppose that $T_{g}: A_{\alpha+}^{p} \rightarrow A_{\beta+}^{q}$ is compact. Since $A_{\alpha+}^{p}$ is a Fréchet-Schwartz space for any fixed $1<p<\infty$ and $0<\alpha<\infty$, the Volterra operator $T_{g}: A_{\alpha+}^{p} \rightarrow A_{\beta+}^{q}$ is bounded. By Lemma 2.5(i), there exists $\varepsilon>0$ such that for $\mu:=\alpha+p \varepsilon$, every $\eta:=\beta+p \delta<\beta+p \varepsilon$ yields $T_{g}: A_{\mu}^{p} \rightarrow A_{\eta}^{q}$ is continuous. If $1+\frac{2+\eta}{q}-\frac{2+\mu}{p}<0$, then by Proposition 2.1(iii), $g$ is constant, so $g \in \mathcal{B}_{a}$, for any $a \in[0, \infty)$. Now let $1+\frac{2+\eta}{q}-\frac{2+\mu}{p} \geq 0$. By Proposition 2.1(i), $g \in \mathcal{B}_{1+\frac{2+\eta}{q}-\frac{2+\mu}{p}}=\mathcal{B}_{\gamma+\frac{p}{q} \delta-\varepsilon}$. Furthermore,

$$
\left|g^{\prime}(z)\right|\left(1-|z|^{2}\right)^{\gamma+\frac{p}{q} \delta-\varepsilon}>\left|g^{\prime}(z)\right|\left(1-|z|^{2}\right)^{\gamma+\delta-\varepsilon} .
$$

Therefore, $g \in \mathcal{B}_{\gamma-\varepsilon+\delta}$. This gives the desired result. For the converse, let there exist $\varepsilon \in(0, \gamma]$ such that $g \in \mathcal{B}_{\gamma-\varepsilon}$. For any $\delta \in(0, \varepsilon]$ we have

$$
\left|g^{\prime}(z)\right|\left(1-|z|^{2}\right)^{\gamma+\frac{p}{q} \delta-\varepsilon}<\left|g^{\prime}(z)\right|\left(1-|z|^{2}\right)^{\gamma-\varepsilon} .
$$

So, $g \in \mathcal{B}_{\gamma+\frac{p}{q} \delta-\varepsilon}$. Then, by Proposition 2.1(i), $T_{g}: A_{\alpha+p \varepsilon}^{p} \rightarrow A_{\beta+p \delta}^{q}$ is continuous. By Lemma 2.5(i), $T_{g}: A_{\alpha+}^{p} \rightarrow A_{\beta+}^{q}$ is compact. In particular, if $\alpha=\beta$ and $p=q$, then part (i) is also true. If suppose that $\frac{2+\beta}{q}<\frac{2+\alpha}{p}$, then by (2.10) part (iii) follows. If we suppose that $\frac{2+\alpha}{p} \leq \frac{2+\beta}{q}$, then (2.11) yields part (ii).

(2) Define $x=\frac{\min \{\alpha, \beta\}+1}{q}$. Let $T_{g}: A_{\alpha-}^{p} \rightarrow A_{\beta-}^{q}$ be compact, equivalently bounded. Then, by Lemma 2.5 (ii), there exists $\varepsilon \in(0, x)$ such that for $-1<\mu:=\beta-q \varepsilon$, every $-1<\alpha-q \varepsilon<\eta<\alpha$ yields $T_{g}: A_{\eta}^{p} \rightarrow A_{\mu}^{q}$ is continuous. If $1+\frac{2+\mu}{q}-\frac{2+\eta}{p}<0$, by Proposition 2.1(iii), $g$ is constant, and there is nothing to prove. Suppose $1+\frac{2+\mu}{q}-\frac{2+\eta}{p} \geq 0$. Note that for any $\delta \in(0, \varepsilon], T_{g}: A_{\alpha-p \delta}^{p} \rightarrow A_{\mu}^{q}$ is continuous. By Proposition 2.1(i), $g \in \mathcal{B}_{\gamma-\varepsilon+\delta}$. Hence, (2.8) is satisfied. For the converse, let there exist $\varepsilon \in(0, x)$ such that for all $\delta \in(0, \varepsilon]$ we have $g \in \mathcal{B}_{\gamma-\varepsilon+\delta}$. Note that

$$
\left|g^{\prime}(z)\right|\left(1-|z|^{2}\right)^{\gamma+\frac{q}{p} \delta-\varepsilon}<\left|g^{\prime}(z)\right|\left(1-|z|^{2}\right)^{\gamma-\varepsilon+\delta} .
$$

This means $g \in \mathcal{B}_{\gamma+\frac{q}{p} \delta-\varepsilon}$. By Proposition 2.1(i), $T_{g}: A_{\alpha-q \delta}^{p} \rightarrow A_{\beta-q \varepsilon}^{q}$ is continuous. By Lemma 2.5(ii), $T_{g}: A_{\alpha-}^{p} \rightarrow A_{\beta-}^{q}$ is compact. In particular, letting $p=q$ and $\alpha=\beta$ yields part (i). If we let $\frac{2+\beta}{q}<\frac{2+\alpha}{p}$, then $g \in \mathcal{B}_{1-\varepsilon+\delta}$, and part (ii) follows. If we take $\frac{2+\alpha}{p} \leq \frac{2+\beta}{q}$, then by (2.12) part (iii) holds. 
Remark 2.7. Let $\gamma:=1+\frac{2+\beta}{q}-\frac{2+\alpha}{p} \geq 0$. Note that by [22, Corollary 7(i)], the Volterra operator $T_{g}: A_{\alpha}^{p} \rightarrow A_{\beta}^{q}$ is compact if and only if $g \in \mathcal{B}_{\gamma}^{0}$. Concerning Proposition 2.6, let $g \in \bigcup_{\tau<\gamma} \mathcal{B}_{\tau}$. So there exists $\varepsilon>0$ with $g \in \mathcal{B}_{\gamma-\varepsilon}$. This means there exists $M>0$ such that $\left|g^{\prime}(z)\right|\left(1-|z|^{2}\right)^{\gamma-\varepsilon} \leq M$, for every $z \in \mathbb{D}$. Let us observe that

$$
\begin{aligned}
\left|g^{\prime}(z)\right|\left(1-|z|^{2}\right)^{\gamma} & =\left|g^{\prime}(z)\right|\left(1-|z|^{2}\right)^{\varepsilon}\left(1-|z|^{2}\right)^{\gamma-\varepsilon} \\
& \leq M\left(1-|z|^{2}\right)^{\varepsilon} \rightarrow 0,
\end{aligned}
$$

as $|z| \rightarrow 1^{-}$. So $g \in \mathcal{B}_{\gamma}^{0}$. Hence $T_{g}: A_{\alpha}^{p} \rightarrow A_{\beta}^{q}$ is compact. Now let $g \in \mathcal{B}_{\gamma}^{0} \backslash \bigcup_{\tau<\gamma} \mathcal{B}_{\tau}$. Then, $T_{g}: A_{\alpha}^{p} \rightarrow A_{\beta}^{q}$ is compact. Suppose that $T_{g}: A_{\alpha+}^{p} \rightarrow A_{\beta+}^{q}$ is compact as well. Then, there exists $\varepsilon \in(0, \gamma]$ such that for $\mu:=\alpha+p \varepsilon$ one has $T_{g}: A_{\mu}^{p} \rightarrow A_{\beta}^{q}$ is continuous. This means,

$$
\infty>\sup _{z \in \mathbb{D}}\left|g^{\prime}(z)\right|\left(1-|z|^{2}\right)^{1+\frac{2+\beta}{q}-\frac{2+\mu}{p}}=\sup _{z \in \mathbb{D}}\left|g^{\prime}(z)\right|\left(1-|z|^{2}\right)^{\gamma-\varepsilon}=\infty .
$$

This is a contradiction.

\section{Spectra of Volterra operators on $A_{\alpha+}^{p}$ and $A_{\alpha-}^{p}$}

Let $E$ be a locally convex Hausdorff space, and $\Gamma_{E}$ a system of continuous seminorms determining the topology of $E$. Let $E^{\prime}$ denote the space of all continuous linear functionals on $E$. Denote the identity operator on $E$ by $I$. Let $\mathcal{L}(E)$ denote the space of all continuous linear operators from $E$ into itself. For $T \in \mathcal{L}(E)$, the resolvent set $\rho(T ; E)$ of $T$ consists of all $\lambda \in \mathbb{C}$ such that $R(\lambda, T):=(\lambda I-T)^{-1}$ exists in $\mathcal{L}(E)$. The set $\sigma(T ; E):=\mathbb{C} \backslash \rho(T)$ is called the spectrum of $T$. The point spectrum $\sigma_{p t}(T ; E)$ of $T$ consists of all $\lambda \in \mathbb{C}$ such that $(\lambda I-T)$ is not injective. Contrary to Banach spaces, concerning the spectrum of an operator $T$ on the Fréchet space $E$, one may encounter that $\rho(T ; E)=\varnothing$ or $\rho(T ; E)$ fails to be an open set in $\mathbb{C}$. For this reason, some authors prefer to consider the subset $\rho^{*}(T ; E)$ of $\rho(T ; E)$ consisting of $\lambda \in \mathbb{C}$ such that there exists $\delta>0$ such that $B(\lambda, \delta):=$ $\{z \in \mathbb{C}:|z-\lambda|<\delta\} \subseteq \rho(T ; E)$ and $\{R(\mu, T): \mu \in B(\lambda, \delta)\}$ is equicontinuous in $\mathcal{L}(E)$. Define the Waelbroeck spectrum $\sigma^{*}(T ; E):=\mathbb{C} \backslash \rho^{*}(T ; E)$, which is a closed set containing $\sigma(T ; E)$. If $T \in \mathcal{L}(E)$ with $E$ a Banach space, then $\sigma^{*}(T ; E)=\sigma(T ; E)$.

Given $\lambda \in \mathbb{C} \backslash\{0\}$, and $h \in H(\mathbb{D})$, the equation $f-\frac{1}{\lambda} T_{g} f=h$ has a unique solution given by

$$
f(z)=R_{\lambda, g} h(z):=h(0) e^{g(z) / \lambda}+e^{g(z) / \lambda} \int_{0}^{z} e^{-g(\zeta) / \lambda} h^{\prime}(\zeta) \mathrm{d} \zeta, \quad z \in \mathbb{D} .
$$

Lemma 3.1 and Lemma 3.2 are taken from [12]. Their proofs are very close to the case of entire functions which were considered in [11].

Lemma 3.1. Let $E$ be a locally convex space continuously contained in $H(\mathbb{D})$ that contains the constants. Assume that $T_{g}: E \rightarrow E$ is continuous for some non-constant entire function $g$ such that $g(0)=0$. Then, the following statements are equivalent:

(i) $\lambda \in \rho\left(T_{g} ; E\right)$.

(ii) $R_{\lambda, g}: E \rightarrow E$ is continuous.

(iii) $e^{g(z) / \lambda} \in E$ and $S_{\lambda, g} h:=e^{g(z) / \lambda} \int_{0}^{z} e^{-g(\zeta) / \lambda} h^{\prime}(\zeta) \mathrm{d} \zeta, z \in \mathbb{C}$ is continuous on the subspace $E_{0}$ of $E$ of all functions $h \in E$ with $h(0)=0$.

In connection with Lemma 3.1, we denote $A_{\alpha}^{p^{0}}$ as the subspace of $A_{\alpha}^{p}$ which stands for functions $h \in A_{\alpha}^{p}$ satisfying $h(0)=0$. Similar notation shall be adapted for $A_{\alpha+}^{p}$ and $A_{\alpha-}^{p}$ throughout. 
Lemma 3.2. Let $E$ be a locally convex space continuously contained in $H(\mathbb{D})$ that contains the constants. Assume that $T_{g}: E \rightarrow E$ is continuous for some non-constant entire function $g$ such that $g(0)=0$. Then,

$$
\{0\} \cup\left\{\lambda \in \mathbb{C} \backslash\{0\}: e^{g / \lambda} \notin E\right\} \subset \sigma\left(T_{g} ; E\right) .
$$

The following abstract spectral results for Fréchet and (LB)-spaces will be useful in proving our assertions.

Lemma 3.3 ([2, Lemma 2.1]). Let $E=\bigcap_{n \in \mathbb{N}} E_{n}$ be a Fréchet space which is the intersection of a sequence of Banach spaces $\left(\left(E_{n},\|\cdot\|_{n}\right)\right)_{n \in \mathbb{N}}$ satisfying $E_{n+1} \subseteq E_{n}$ with $\|x\|_{n} \leq\|x\|_{n+1}$, for all $n \in \mathbb{N}$ and $x \in E_{n+1}$. Let $T \in \mathcal{L}(E)$. Suppose that for all $n \in \mathbb{N}$, there exists $T_{n} \in \mathcal{L}\left(E_{n}\right)$ such that the restriction of $T_{n}$ to $E$ (resp. of $T_{n}$ to $E_{n+1}$ ) coincides with $T$ (resp. $\left.T_{n+1}\right)$. Then, the following statements hold:

(i) $\sigma(T ; E) \subseteq \cup_{n \in \mathbb{N}} \sigma\left(T_{n} ; E_{n}\right)$ and $R(\lambda, T)$ coincides with the restriction of $R\left(\lambda, T_{n}\right)$ to $E$, for all $n \in \mathbb{N}$ and each $\lambda \in \bigcap_{n \in \mathbb{N}} \rho\left(T_{n} ; E_{n}\right)$.

(ii) If $\cup_{n \in \mathbb{N}} \sigma\left(T_{n} ; E_{n}\right) \subseteq \overline{\sigma(T ; E)}$, then

$$
\sigma^{*}(T ; E)=\overline{\sigma(T ; E)} .
$$

Lemma 3.4 ([3, Lemma 5.2]). Let $E=\operatorname{ind}_{n \in \mathbb{N}}\left(E_{n},\|\cdot\|_{n}\right)$ be a Hausdorff inductive limit of Banach spaces. Let $T \in \mathcal{L}(E)$. Suppose that for each $n \in \mathbb{N}$, the restriction $T_{n}$ of $T$ to $E_{n}$ maps $E_{n}$ into itself and $T_{n} \in \mathcal{L}\left(E_{n}\right)$. Then, the following properties are satisfied:

(i) $\sigma_{p t}(T ; E)=\bigcup_{n \in \mathbb{N}} \sigma_{p t}\left(T_{n} ; E_{n}\right)$.

(ii) $\sigma(T ; E) \subseteq \bigcap_{m \in \mathbb{N}}\left(\bigcup_{n=m}^{\infty} \sigma\left(T_{n} ; E_{n}\right)\right)$. Moreover, if $\lambda \in \bigcap_{n=m}^{\infty} \rho\left(T_{n} ; E_{n}\right)$ for some $m \in \mathbb{N}$, then $R\left(\lambda, T_{n}\right)$ coincides with the restriction of $R(\lambda, T)$ to $E_{n}$, for every $n \geq m$.

(iii) If $\bigcup_{n=m}^{\infty} \sigma\left(T_{n} ; E_{n}\right) \subseteq \overline{\sigma(T ; E)}$, for some $m \in \mathbb{N}$, then

$$
\sigma^{*}(T ; E)=\overline{\sigma(T ; E)} \text {. }
$$

For every $z \in \mathbb{D}$, we define the Möbius transformation

$$
\varphi_{z}(w):=\frac{z-w}{1-\bar{z} w}, \quad w \in \mathbb{D} .
$$

It is routine to check that the change of variables $u=\varphi_{z}(w)$ gives

$$
\begin{gathered}
1-|u|^{2}=\frac{\left(1-|z|^{2}\right)\left(1-|w|^{2}\right)}{|1-\bar{z} w|^{2}}, \quad J_{u}=\frac{\left(1-|z|^{2}\right)^{2}}{|1-\bar{z} w|^{4}}, \\
\text { and } \quad|1-\bar{z} w|=\frac{1-|z|^{2}}{|1-\bar{z} u|} .
\end{gathered}
$$

where $J_{u}$ is the real Jacobian term. For $1<p<\infty$, and $-1<\alpha<\infty$ we have the relation

$$
\|f\|_{p, \alpha} \asymp\left\|f^{\prime}\right\|_{p, p+\alpha}+|f(0)|, \quad \forall f \in H(\mathbb{D}) .
$$

One inequality is a classical result due to Hardy-Littlewood [14, Theorem 5.6], and the reverse inequality is easily proved by the methods in [14, Chapter 5]. Now we state our first result concerning the spectrum of the Volterra operator. 
Proposition 3.5. Let $1<p<\infty$, and $0<\alpha<\infty$. If $g \in \mathcal{B}_{1}$, then

(1) The spectrum of Volterra operator $T_{g}: A_{\alpha+}^{p} \rightarrow A_{\alpha+}^{p}$ satisfies

$$
\sigma\left(T_{g} ; A_{\alpha+}^{p}\right)=\bigcup_{\mu>\alpha} \sigma\left(T_{g} ; A_{\mu}^{p}\right) .
$$

(2) The spectrum of Volterra operator $T_{g}: A_{\alpha-}^{p} \rightarrow A_{\alpha-}^{p}$ satisfies

$$
\sigma\left(T_{g} ; A_{\alpha-}^{p}\right)=\bigcap_{\mu<\alpha}\left(\bigcup_{\eta \in(\mu, \alpha)} \sigma\left(T_{g} ; A_{\eta}^{p}\right)\right) .
$$

Proof. (1) One inclusion follows by Lemma 3.3. For the other inclusion, assume that $\lambda \notin \sigma\left(T_{g} ; A_{\alpha+}^{p}\right)$. Then, by Lemma $3.1 e^{g / \lambda} \in A_{\mu}^{p}$, for every $\mu>\alpha$. We show $\lambda \notin$ $\bigcup_{\mu>\alpha} \sigma\left(T_{g} ; A_{\mu}^{p}\right)$. Suppose not. Then, there exists $\beta>\alpha$ such that $\lambda \in \sigma\left(T_{g} ; A_{\beta}^{p}\right)$. Applying Lemma 3.1 once again for $A_{\beta}^{p}$, we see that $S_{\lambda, g} \notin \mathcal{L}\left(A_{\beta}^{p^{0}}\right)$. On the other hand, using the change of variables $u=\varphi_{z}(\zeta)$ for an arbitrary $z \in \mathbb{D}$, by (3.1) we obtain

$$
\begin{aligned}
\infty & >\left\|e^{g / \lambda}\right\|_{p, \beta}^{p}=(\beta+1) \int_{\mathbb{D}}\left|e^{\frac{g(u)}{\lambda}}\right|^{p}\left(1-|u|^{2}\right)^{\beta} \mathrm{d} s(u) \\
& \asymp \int_{\mathbb{D}}\left|e^{\frac{g(\zeta)}{\lambda}}\right|^{p}\left(\frac{1-|z|^{2}}{|1-\bar{z} \zeta|^{2}}\right)^{2+\beta}\left(1-|\zeta|^{2}\right)^{\beta} \mathrm{d} s(\zeta) .
\end{aligned}
$$

Then, by [13, Theorem 1], the multiplication operator $M_{e^{g / \lambda}}$ is continuous on $A_{\beta}^{p}$. Thus, $\omega(z):=e^{p \operatorname{Re} \frac{g(z)}{\lambda}}\left(1-|z|^{2}\right)^{\beta}$ is a weight function in $\mathbb{D}$, and as illustrated in [5, p. 202] the weighted Bergman space norm induced by $\omega$ satisfies the property $\left\|e^{-g / \lambda} S_{\lambda, g} h\right\|_{p, \omega} \asymp$ $\left\|\left(e^{-g / \lambda} S_{\lambda, g} h\right)^{\prime}\right\|_{p, \hat{\omega}}=\left\|e^{-g / \lambda} h^{\prime}\right\|_{p, \hat{\omega}}$, for $h \in A_{\beta}^{p^{0}}$ if we denote $\hat{\omega}(z)=\omega(z)\left(1-|z|^{2}\right)^{p}$. But then, for every $h \in A_{\beta}^{p^{0}}$ we obtain by (3.2) that (cf. [5, Theorem 5.1])

$$
\begin{aligned}
\left\|S_{\lambda, g} h\right\|_{p, \beta}^{p} & =\left\|\frac{1}{e^{g / \lambda}} S_{\lambda, g} h\right\|_{p, \omega}^{p} \asymp\left\|\left(\frac{1}{e^{g / \lambda}} S_{\lambda, g} h\right)^{\prime}\right\|_{p, \hat{\omega}}^{p}=\left\|\frac{1}{e^{g / \lambda}} h^{\prime}\right\|_{p, \hat{\omega}}^{p} \\
& =(\beta+p+1) \int_{\mathbb{D}}\left|h^{\prime}(z)\right|^{p}\left(1-|z|^{2}\right)^{\beta+p} \mathrm{~d} s(z) \\
& =\left\|h^{\prime}\right\|_{p, \beta+p} \asymp\|h\|_{p, \beta}^{p},
\end{aligned}
$$

which implies that $S_{\lambda, g}$ is continuous on $A_{\beta}^{p^{0}}$. This is a contradiction.

(2) Similarly, one of the inclusions follow by Lemma 3.4. For the other inclusion, assume that $\lambda \notin \sigma\left(T_{g} ; A_{\alpha-}^{p}\right)$. We show $\lambda \notin \bigcap_{\mu<\alpha}\left(\bigcup_{\eta \in(\mu, \alpha)} \sigma\left(T_{g} ; A_{\eta}^{p}\right)\right)$. Assume contrary. Then, for every $\mu<\alpha$ there exists $\eta \in(\mu, \alpha)$ such that $\lambda \in \sigma\left(T_{g} ; A_{\eta}^{p}\right)$. Since $\lambda \in \rho\left(T_{g} ; A_{\alpha-}^{p}\right)$, by Lemma 3.1 we have $e^{g / \lambda} \in A_{\alpha-}^{p}$ and so there exists $\beta<\alpha$ such that $e^{g / \lambda} \in A_{\mu}^{p}$ for every $\mu \in[\beta, \alpha)$. Without loss of any generality, suppose $\eta \geq \beta$. The rest is very similar to part $(1)$.

Given an analytic function $g \in \mathbb{D}$, we denote by $g_{z}, z \in \mathbb{D}$ its hyperbolic translate $g_{z}(\zeta):=g\left(\varphi_{z}(\zeta)\right)-g(z), \zeta \in \mathbb{D}$. The following important result shall be quoted for several occasions. 
Theorem 3.6 ([5, Theorem A; B; 5.1]). Let $p>0$ and let $\alpha>-1$. Let $g \in \mathcal{B}_{1}$. Then, the following statements hold:

(i) $\sigma_{p t}\left(T_{g} ; A_{\alpha}^{p}\right)=\varnothing$.

(ii) For every $\gamma>-2 \alpha-3$, the spectrum of the Volterra operator $\sigma\left(T_{g} ; A_{\alpha}^{p}\right)$ includes

$$
\{0\} \cup\left\{\lambda \in \mathbb{C}: \sup _{z \in \mathbb{D}} \int_{\mathbb{D}} e^{p \operatorname{Re} \frac{g_{z}(\zeta)}{\lambda}}|1-\bar{z} \zeta|^{\gamma}\left(1-|\zeta|^{2}\right)^{\alpha} \mathrm{d} s(\zeta)=\infty\right\} .
$$

If $g^{\prime}$ is rational, then this condition is equivalent to $e^{g / \lambda} \notin A_{\alpha}^{p}$.

(iii) There exists $g \in \mathcal{B}_{1}$ such that $e^{g / \lambda} \in A_{\alpha}^{p}$, for all $\lambda \in \mathbb{C} \backslash\{0\}$ but

$$
\sigma\left(T_{g} ; A_{\alpha}^{p}\right) \supset\left\{\lambda \in \mathbb{C}:\left|\lambda-\frac{p}{2(2+\alpha)}\right| \leq \frac{p}{2(2+\alpha)}\right\} .
$$

(iv) If $g^{\prime}(z)=\int_{\mathbb{T}} \frac{\mathrm{d} \mu(\zeta)}{\zeta-z}, z \in \mathbb{D}$ for some finite measure $\mu$ on the unit circle,

$$
\begin{aligned}
\sigma\left(T_{g} ; A_{\alpha}^{p}\right) & =\{0\} \cup \overline{\left\{\lambda \in \mathbb{C} \backslash\{0\}: e^{g / \lambda} \notin A_{\alpha}^{p}\right\}} \\
& =\{0\} \cup\left\{\lambda \in \mathbb{C} \backslash\{0\}: \sup _{\zeta \in \mathbb{T}} \operatorname{Re} \frac{\mu(\{\zeta\})}{\lambda} \geq \frac{2+\alpha}{p}\right\} .
\end{aligned}
$$

Theorem 3.7. Let $1<p<\infty$, and $0<\alpha<\infty$. Let $g \in \mathcal{B}_{1}$. Then, the following statements hold.

(1) $\sigma_{p t}\left(T_{g} ; A_{\alpha+}^{p}\right)=\varnothing$.

(2) For every $\gamma \geq-2 \alpha-3$, the set $\sigma\left(T_{g} ; A_{\alpha+}^{p}\right)$ contains (3.3).

(3) There exists $g \in \mathcal{B}_{1}$ such that $e^{g / \lambda} \in A_{\alpha+}^{p}$, for all $\lambda \in \mathbb{C} \backslash\{0\}$ but

$$
\sigma\left(T_{g} ; A_{\alpha+}^{p}\right) \supset\{0\} \cup\left\{\lambda \in \mathbb{C}:\left|\lambda-\frac{p}{2(2+\alpha)}\right|<\frac{p}{2(2+\alpha)}\right\} .
$$

(4) If $g^{\prime}(z)=\int_{\mathbb{T}} \frac{\mathrm{d} \mu(\zeta)}{\zeta-z}, z \in \mathbb{D}$ for some finite measure $\mu$ on the unit circle,

(i) The spectrum of the Volterra operator on $A_{\alpha+}^{p}$ reads as

$$
\begin{aligned}
\sigma\left(T_{g} ; A_{\alpha+}^{p}\right) & =\{0\} \cup\left\{\lambda \in \mathbb{C} \backslash\{0\}: \operatorname{Re} \frac{\mu(\{\zeta\})}{\lambda}>\frac{2+\alpha}{p}\right\} \\
& =\{0\} \cup\left\{\lambda \in \mathbb{C} \backslash\{0\}: e^{g / \lambda} \notin A_{\alpha+}^{p}\right\} .
\end{aligned}
$$

(ii) $\sigma^{*}\left(T_{g} ; A_{\alpha+}^{p}\right)=\overline{\sigma\left(T_{g} ; A_{\alpha+}^{p}\right)}$.

Proof. (1) Let $T_{g}: A_{\alpha+}^{p} \rightarrow A_{\alpha+}^{p}$ have an eigenvalue $\lambda \in \mathbb{C}$. Suppose that $f \in A_{\alpha+}^{p} \backslash\{0\}$ is its corresponding eigenvector. But then, there exists $\mu>\alpha$ with $f \in A_{\mu}^{p}$ such that $T_{g} f=\lambda f$ in $A_{\mu}^{p}$ hence $\lambda \in \sigma_{p t}\left(T_{g} ; A_{\mu}^{p}\right)$. This contradicts Theorem 3.6(i).

(2) By Lemma 3.2 we directly have $0 \in \sigma\left(T_{g} ; A_{\alpha+}^{p}\right)$. By Proposition 3.5 and Theorem 3.6(ii) we already know that for every $\gamma>-2 \mu-3$ we have

$$
\begin{aligned}
& \sigma\left(T_{g} ; A_{\alpha+}^{p}\right)=\bigcup_{\mu>\alpha} \sigma\left(T_{g} ; A_{\mu}^{p}\right) \\
& \supset \bigcup_{\mu>\alpha}\left\{\lambda \in \mathbb{C}: \sup _{z \in \mathbb{D}} \int_{\mathbb{D}} e^{p \operatorname{Re} \frac{g_{z}(\zeta)}{\lambda}}|1-\bar{z} \zeta|^{\gamma}\left(1-|\zeta|^{2}\right)^{\mu} \mathrm{d} s(\zeta)=\infty\right\} .
\end{aligned}
$$

We show that the latter includes (3.3). Take any $\lambda \in \mathbb{C} \backslash\{0\}$ satisfying the equation in (3.3), and assume contrary. Then, for every $\varepsilon>0$, given $\mu:=\alpha+\varepsilon$ there exist $\gamma_{\mu}>-2 \mu-3$ and $c_{\mu}>0$ such that

$$
\sup _{z \in \mathbb{D}} \int_{\mathbb{D}} e^{p \operatorname{Re} \frac{g_{z}(\zeta)}{\lambda}}|1-\bar{z} \zeta|^{\gamma_{\mu}}\left(1-|\zeta|^{2}\right)^{\mu} \mathrm{d} s(\zeta)<c_{\mu} .
$$


Since $\gamma_{\mu}+2 \varepsilon>-2 \alpha-3$, by (3.1), the change of variables $w=\varphi_{z}(\zeta)$ yields

$$
\begin{aligned}
\infty & =\sup _{z \in \mathbb{D}} \int_{\mathbb{D}} e^{p \operatorname{Re} \frac{g z(\zeta)}{\lambda}}|1-\bar{z} \zeta|^{\gamma_{\mu}+2 \varepsilon}\left(1-|\zeta|^{2}\right)^{\alpha} \mathrm{d} s(\zeta) \\
& =\sup _{z \in \mathbb{D}} \frac{\left(1-|z|^{2}\right)^{\alpha+2+\gamma_{\mu}+2 \varepsilon}}{e^{p \operatorname{Re} \frac{g(z)}{\lambda}}} \int_{\mathbb{D}} e^{p \operatorname{Re} \frac{g(w)}{\lambda}} \frac{\left(1-|w|^{2}\right)^{\alpha}}{|1-\bar{z} w|^{2 \alpha+4+\gamma_{\mu}+2 \varepsilon}} \frac{\left(1-|w|^{2}\right)^{\varepsilon}}{\left(1-|w|^{2}\right)^{\varepsilon}} \mathrm{d} s(w) \\
& \leq \sup _{z \in \mathbb{D}} \frac{\left(1-|z|^{2}\right)^{\mu+2+\gamma_{\mu}}}{e^{p \operatorname{Re} \frac{g(z)}{\lambda}}} \int_{\mathbb{D}} e^{p \operatorname{Re} \frac{g(w)}{\lambda}} \frac{\left(1-|w|^{2}\right)^{\mu}}{|1-\bar{z} w|^{2 \mu+4+\gamma_{\mu}}} \mathrm{d} s(w) .
\end{aligned}
$$

By the change of variables $u=\varphi_{z}(w)$ we obtain

$$
\sup _{z \in \mathbb{D}} \int_{\mathbb{D}} e^{p \operatorname{Re} \frac{g_{z}(u)}{\lambda}}|1-\bar{z} u|^{\gamma_{\mu}}\left(1-|u|^{2}\right)^{\mu} \mathrm{d} s(u)=\infty .
$$

That contradicts (3.4).

(3) Let us choose $g \in \mathcal{B}_{1}$ as in Theorem 3.6(iii). Since $A_{\alpha}^{p} \subseteq A_{\alpha+}^{p}$, we obtain $e^{g / \lambda} \in A_{\alpha+}^{p}$, for all $\lambda \in \mathbb{C} \backslash\{0\}$. We know by Lemma 3.2 that $0 \in \sigma\left(T_{g} ; A_{\alpha+}^{p}\right)$. Now let us take $\lambda \in \mathbb{C} \backslash\{0\}$ with $\left|\lambda-\frac{p}{2(2+\alpha)}\right|<\frac{p}{2(2+\alpha)}$. Then, there exists $N \in \mathbb{N}$ such that $\left|\lambda-\frac{p}{2\left(2+\left(\alpha+\frac{1}{n}\right)\right)}\right| \leq$ $\frac{p}{2\left(2+\left(\alpha+\frac{1}{n}\right)\right)}$, for all $n \geq N$. Suppose that $\lambda \in \rho\left(T_{g} ; A_{\alpha+}^{p}\right)$. By Theorem 3.6(iii), $\lambda \in$ $\sigma\left(T_{g} ; A_{\left(\alpha+\frac{1}{n}\right)}^{p}\right)$, for all $n \geq N$. On the other hand, by Proposition 3.5, we have $\lambda \in$ $\bigcap_{n \in \mathbb{N}} \rho\left(T_{g} ; A_{\left(\alpha+\frac{1}{n}\right)}^{p}\right)$. This is a contradiction.

(4) By Proposition 3.5 and Theorem 3.6 (iv),

$$
\begin{aligned}
\sigma\left(T_{g} ; A_{\alpha+}^{p}\right) & =\bigcup_{n \in \mathbb{N}} \sigma\left(T_{g} ; A_{\left(\alpha+\frac{1}{n}\right)}^{p}\right) \\
& =\{0\} \cup \bigcup_{n \in \mathbb{N}}\left\{\lambda \in \mathbb{C} \backslash\{0\}: \operatorname{Re} \frac{\mu(\{\zeta\})}{\lambda} \geq \frac{2+\left(\alpha+\frac{1}{n}\right)}{p}\right\} \\
& =\{0\} \cup\left\{\lambda \in \mathbb{C} \backslash\{0\}: \operatorname{Re} \frac{\mu(\{\zeta\})}{\lambda}>\frac{2+\alpha}{p}\right\} .
\end{aligned}
$$

For the second equality suppose $\lambda \in \sigma\left(T_{g} ; A_{\alpha+}^{p}\right)$. Then by Lemma 3.3(i) $\lambda \in \cup_{\mu>\alpha} \sigma\left(T_{g} ; A_{\mu}^{p}\right)$, hence there exists $\beta>\alpha$ with $\lambda \in \sigma\left(T_{g} ; A_{\beta}^{p}\right)$. So by Theorem 3.6(iv), we obtain $e^{g / \lambda} \notin A_{\beta}^{p}$. Hence $e^{g / \lambda} \notin A_{\alpha+}^{p}$. Thus, $\sigma\left(T_{g} ; A_{\alpha+}^{p}\right) \subset\{0\} \cup\left\{\lambda \in \mathbb{C} \backslash\{0\}: e^{g / \lambda} \notin A_{\alpha+}^{p}\right\}$. Combining with Lemma 3.2 we prove that (i) holds. For part (ii), thanks to Lemma 3.3(ii), it suffices to make sure that $\bigcup_{\mu>\alpha} \sigma\left(T_{g} ; A_{\mu}^{p}\right) \subseteq \overline{\sigma\left(T_{g} ; A_{\alpha+}^{p}\right)}$. This is true by part (i).

Theorem 3.8. Let $1<p<\infty$, and $0<\alpha<\infty$. Let $g \in \mathcal{B}_{1}$. Then, the following statements hold.

(1) $\sigma_{p t}\left(T_{g} ; A_{\alpha-}^{p}\right)=\varnothing$.

(2) For every $\gamma>-2 \alpha-3$, the set $\sigma\left(T_{g} ; A_{\alpha-}^{p}\right)$ contains (3.3).

(3) There exists $g \in \mathcal{B}_{1}$ such that $e^{g / \lambda} \in A_{\alpha-}^{p}$, for all $\lambda \in \mathbb{C} \backslash\{0\}$ but

$$
\sigma\left(T_{g} ; A_{\alpha-}^{p}\right) \supset\left\{\lambda \in \mathbb{C}:\left|\lambda-\frac{p}{2(2+\alpha)}\right| \leq \frac{p}{2(2+\alpha)}\right\} .
$$

(4) If $g^{\prime}(z)=\int_{\mathbb{T}} \frac{\mathrm{d} \mu(\zeta)}{\zeta-z}, z \in \mathbb{D}$ for some finite measure $\mu$ on the unit circle,

(i) The spectrum of $T_{g}$ on $A_{\alpha-}^{p}$ reads

$$
\sigma\left(T_{g} ; A_{\alpha-}^{p}\right)=\{0\} \cup\left\{\lambda \in \mathbb{C} \backslash\{0\}: \sup _{\zeta \in \mathbb{T}} \operatorname{Re} \frac{\mu(\{\zeta\})}{\lambda} \geq \frac{2+\alpha}{p}\right\} .
$$

(ii) $\sigma^{*}\left(T_{g} ; A_{\alpha-}^{p}\right)=\sigma\left(T_{g} ; A_{\alpha-}^{p}\right)$. 
Proof. (1) This is a direct consequence of Lemma 3.4 and Theorem 3.6(i).

(2) By Proposition 3.5 and Theorem 3.6(ii), for every $\gamma>-2 \eta-3$ we have

$$
\begin{aligned}
& \sigma\left(T_{g} ; A_{\alpha-}^{p}\right) \\
& =\bigcap_{\mu<\alpha}\left(\bigcup_{\eta \in(\mu, \alpha)} \sigma\left(T_{g} ; A_{\eta}^{p}\right)\right) \\
& \supset \bigcap_{\mu<\alpha} \bigcup_{\eta \in(\mu, \alpha)}\left\{\lambda \in \mathbb{C}: \sup _{z \in \mathbb{D}} \int_{\mathbb{D}} e^{p \operatorname{Re} \frac{g_{z}(\zeta)}{\lambda}}|1-\bar{z} \zeta|^{\gamma}\left(1-|\zeta|^{2}\right)^{\eta} \mathrm{d} s(\zeta)=\infty\right\} .
\end{aligned}
$$

We show that the latter includes (3.3). So we take any $\lambda \in \mathbb{C} \backslash\{0\}$ satisying the equality in (3.3), and assume contrary. Then, there exists $-1<\mu:=\alpha-\varepsilon$ such that for every $\mu<\eta:=\alpha-\delta<\alpha$ there are $\gamma_{\eta}>-2 \eta-3$ and $c_{\eta}>0$ satisfying

$$
\sup _{z \in \mathbb{D}} \int_{\mathbb{D}} e^{p \operatorname{Re} \frac{g_{z}(\zeta)}{\lambda}}|1-\bar{z} \zeta|^{\gamma_{\eta}}\left(1-|\zeta|^{2}\right)^{\eta} \mathrm{d} s(\zeta)<c_{\eta} .
$$

Note that for every $z, \zeta \in \mathbb{D}$ we have

$$
\frac{1}{|1-\bar{z} \zeta|} \leq \frac{1}{1-|\zeta|} \leq \frac{2}{1-|\zeta|^{2}}
$$

Since we have $\gamma_{\eta}-\delta>-2 \alpha-3$, we obtain

$$
\begin{aligned}
\infty & =\sup _{z \in \mathbb{D}} \int_{\mathbb{D}} e^{p \operatorname{Re} \frac{g_{z}(\zeta)}{\lambda}}|1-\bar{z} \zeta|^{\gamma_{\eta}-\delta}\left(1-|\zeta|^{2}\right)^{\alpha} \mathrm{d} s(\zeta) \\
& \leq 2 \sup _{z \in \mathbb{D}} \int_{\mathbb{D}} e^{p \operatorname{Re} \frac{g_{z}(\zeta)}{\lambda}}|1-\bar{z} \zeta|^{\gamma_{\eta}}\left(1-|\zeta|^{2}\right)^{\eta} \mathrm{d} s(\zeta)<c_{\eta} .
\end{aligned}
$$

This is a contradiction.

(3) In the selection of $g \in \mathcal{B}_{1}$, we follow Theorem 3.6(iii). In [5, p. 229] it is shown that with this particular choice of $g$, the function $e^{g / \lambda}$ belongs to the Hardy space $H^{p}$, for every $p>0$. In particular, for every $\lambda \in \mathbb{C} \backslash\{0\}$ we have $e^{g / \lambda} \in A_{\left(\alpha-\frac{1}{n}\right)}^{p}$, for all $n \in \mathbb{N}$. Let us take $\lambda \in \mathbb{C} \backslash\{0\}$ satisfying $\left|\lambda-\frac{p}{2(2+\alpha)}\right| \leq \frac{p}{2(2+\alpha)}$ and suppose that $\lambda \in \rho\left(T_{g} ; A_{\alpha-}^{p}\right)$. It is clear that for any $n \in \mathbb{N}$ we have $\left|\lambda-\frac{p}{2\left(2+\left(\alpha-\frac{1}{n}\right)\right)}\right| \leq \frac{p}{2\left(2+\left(\alpha-\frac{1}{n}\right)\right)}$ and hence by Theorem 3.6(iii) we obtain $\lambda \in \sigma\left(T_{g} ; A_{\left(\alpha-\frac{1}{n}\right)}^{p}\right)$, for any $n \in \mathbb{N}$. By Proposition 3.5(2), this is a contradiction.

(4) By Proposition 3.5 and Theorem 3.6(iv) we obtain

$$
\begin{aligned}
\sigma\left(T_{g} ; A_{\alpha-}^{p}\right) & =\bigcap_{m \in \mathbb{N}} \bigcup_{n \geq m} \sigma\left(T_{g} ; A_{\left(\alpha-\frac{1}{n}\right)}^{p}\right) \\
& =\{0\} \cup \bigcap_{m \in \mathbb{N}} \bigcup_{n \geq m}\left\{\lambda \in \mathbb{C} \backslash\{0\}: \sup _{\zeta \in \mathbb{T}} \operatorname{Re} \frac{\mu(\{\zeta\})}{\lambda} \geq \frac{2+\left(\alpha-\frac{1}{n}\right)}{p}\right\} \\
& =\{0\} \cup \bigcap_{m \in \mathbb{N}}\left\{\lambda \in \mathbb{C} \backslash\{0\}: \sup _{\zeta \in \mathbb{T}} \operatorname{Re} \frac{\mu(\{\zeta\})}{\lambda}>\frac{2+\left(\alpha-\frac{1}{m}\right)}{p}\right\} \\
& =\{0\} \cup\left\{\lambda \in \mathbb{C} \backslash\{0\}: \sup _{\zeta \in \mathbb{T}} \operatorname{Re} \frac{\mu(\{\zeta\})}{\lambda} \geq \frac{2+\alpha}{p}\right\} .
\end{aligned}
$$

This proves part (i). For part (ii), let us adapt the argument from [3, Proposition 2.9] (also used in [12, Proposition 3.9] and [17, Theorem 2.7(3)]). Let $\lambda \notin \sigma\left(T_{g} ; A_{\alpha-}^{p}\right)$. 
Then there exist $r>0$ and $N \in \mathbb{N}$ such that for every $n \geq N$,

$$
\overline{B(\lambda, r)} \cap\left(\{0\} \cup\left\{\lambda \in \mathbb{C} \backslash\{0\}: \sup _{\zeta \in \mathbb{T}} \operatorname{Re} \frac{\mu(\{\zeta\})}{\lambda} \geq \frac{2+\alpha}{p}\right\}\right)=\varnothing .
$$

Therefore, $\overline{B(\lambda, r)} \subset \rho\left(T_{g} ; A_{\alpha-}^{p}\right)$, as well as $\overline{B(\lambda, r)} \subset \rho\left(T_{g} ; A_{\left(\alpha-\frac{1}{n}\right)}^{p}\right)$, for all $n \geq N$. In particular, the set $\left\{R\left(\delta, T_{g}\right): \delta \in \overline{B(\lambda, r)}\right\}$ is equicontinuous, that is, operator norm bounded in $\mathcal{L}\left(A_{\left(\alpha-\frac{1}{n}\right)}^{p}\right)$, for all $n \geq N$. It remains to show that $\left\{R\left(\delta, T_{g}\right): \delta \in \overline{B(\lambda, r)}\right\}$ is equicontinuous in $A_{\alpha-}^{p}$. Since $A_{\alpha-}^{p}$ is a (DFS)-space, we can apply Banach-Steinhaus principle. So it suffices to show that $\left\{R\left(\delta, T_{g}\right) f: \delta \in \overline{B(\lambda, r)}\right\}$ is a bounded set in $A_{\alpha-}^{p}$, for every $f \in A_{\alpha-}^{p}$. Let us fix $f \in A_{\alpha-}^{p}$. Then, $f \in A_{\left(\alpha-\frac{1}{n}\right)}^{p}$, for some $n \geq N$. So $\left\{R\left(\delta, T_{g}\right) f: \delta \in \overline{B(\lambda, r)}\right\}$ is a bounded set in $A_{\left(\alpha-\frac{1}{n}\right)}^{p}$, and hence in $A_{\alpha-}^{p}$.

Acknowledgment. This article was completed during the author's stay at Instituto Universitario de Matemática Pura y Aplicada, Universitat Politècnica de València, funded by The Scientific and Technological Research Council of Turkey International Postdoctoral Research Fellowship (TÜBİTAK 2219) with grant number 1059B191800828. The author is deeply thankful to Prof. José Bonet, Prof. Enrique Jordá, and Prof. David Jornet for useful suggestions and kind hospitality.

\section{References}

[1] A. Albanese, J. Bonet and W.J. Ricker, The Cesàro operator in growth Banach spaces of analytic functions, Integr. Equ. Oper. Theory 86, 97-112, 2016.

[2] A. Albanese, J. Bonet and W. Ricker, The Cesàro operator in the Fréchet spaces $\ell^{p+}$ and $L^{p-}$, Glasg. Math. J. 59 (2), 273-287, 2017.

[3] A. Albanese, J. Bonet and W. Ricker, The Cesàro operator on Korenblum type spaces of analytic functions, Collect. Math. 69 (2), 263-281, 2018.

[4] A. Aleman and J.A. Cima, An integral operator on $H^{p}$ and Hardy's inequality, J. Anal. Math. 85, 157-176, 2001.

[5] A. Aleman and O. Constantin, Spectra of integration operators on weighted Bergman spaces, J. Anal. Math. 109, 199-231, 2009.

[6] A. Aleman and J.A. Peláez, Spectra of integration operators and weighted square functions, Indiana Univ. Math. J. 61, 1-19, 2012.

[7] A. Aleman and A. Persson, Resolvent estimates and decomposable extensions of generalized Cesàro operators, J. Funct. Anal. 258, 67-98, 2010.

[8] A. Aleman and A.G. Siskakis, An integral operator on $H^{p}$, Complex Var. Theory Appl. 28, 149-158, 1995.

[9] A. Aleman and A.G. Siskakis, Integration operators on Bergman spaces, Indiana Univ. Math. J. 46, 337-356, 1997.

[10] M. Basallote, M.D. Contreras, C. Hernández-Mancera, M.J. Martín and P.J. Paúl, Volterra operators and semigroups in weighted Banach spaces of analytic functions, Collect. Math. 65, 233-249, 2014.

[11] J. Bonet, The spectrum of Volterra operators on weighted spaces of entire functions, Quart. J. Math. 66, 799-807, 2015.

[12] J. Bonet, The spectrum of Volterra operators on Korenblum type spaces of analytic functions, Integr. Equ. Oper. Theory 91, 46, 2019.

[13] Ž. Čučković and R. Zhao, Weighted composition operators between different weighted Bergman spaces and different Hardy spaces, Ilinois J. Math. 51 (2), 479-498, 2007.

[14] P. Duren, Theory of $H^{p}$ Spaces, Academic Press, New York, 1970. 
[15] H. Hedenmalm, B. Korenblum and K. Zhu, Theory of Bergman Spaces, Graduate Texts in Math. 199, Springer, New York, 2000.

[16] H. Jarchow, Locally Convex Spaces, Teubner, Stuttgart, 1981.

[17] E. Kizgut, The Cesàro operator on weighted Bergman Fréchet and (LB)-spaces of analytic functions, Filomat, to appear.

[18] R. Korhonen and J. Rättyä, Intersections and unions of weighted Bergman spaces, Comput. Methods Funct. Theory 5 (2), 459-469, 2005.

[19] B. Malman, Spectra of generalized Cesàro operators acting on growth spaces, Integr. Equ. Oper. Theory 90, 26, 2018.

[20] R. Meise and D. Vogt, Introduction to Functional Analysis, Oxford Graduate Texts in Mathematics, Clarendon Press, Oxford, 1997.

[21] C. Pommerenke, Schlichte Funktionen un analytische Funktionen von beschränkter mittlerer Oszilation, Comment. Math. Helv. 52, 591-602, 1977.

[22] J. Rättyä, Integration operator acting on Hardy and weighted Bergman spaces, Bull. Aust. Math. Soc. 75, 431-446, 2006.

[23] A. Siskakis, Volterra operators on spaces of analytic functions-a survey, in: Proceedings of the First Advanced Course in Operator Theory and Complex Analysis, 51-68, Univ. Sevilla Serc. Publ., Seville, 2006.

[24] K. Zhu, Operator Theory on Function Spaces, Mathematical surveys and monographs 138, American Mathematical Society, 2007. 